\title{
Intensity discrimination for pulsed sinusoids of various frequencies*
}

\author{
M. J. PENNER \\ Bell Telephone Laboratories, Holmdel, New Jersey 07733 \\ and \\ B. LESHOWITZ, E. CUDAHY, and G. RICARD \\ Arizona State University, Tempe, Arizona 85281
}

\begin{abstract}
Studies of pure-tone intensity discrimination have shown that Weber's law fails for tones in the region of $1 \mathrm{kHz}$. In this experiment, intensity discrimination of pulsed sinusoids ranging in frequency from 0.15 to $12 \mathrm{kHz}$ is investigated. For each tone in this region, Weber's law is found to fail. Some theoretical implications of these results are discussed.
\end{abstract}

Weber's law does not hold for intensity discrimination of pulsed tones in the region of $1 \mathrm{kHz}$ (Campbell \& Lasky, 1967; McGill \& Goldberg, 1968a, b; Viemeister, 1972). Instead, the masking function $(\Delta I$ in $d B$ as a function of $I$ in $d B$ ) is a straight line with a slope of approximately 0.9 . Thus, intensity discrimination improves at high levels (i.e., the Weber fraction decreases). The improvement has been termed the "near-miss" to Weber's law and has motivated several theoretical explanations (Zwicker, 1965; McGill \& Goldberg, 1968a, b; Viemeister, 1972). The validity of the explanations can be tested by applying them to data on intensity discrimination of sinusoids in other frequency regions. Thus, in this experiment, intensity discrimination of pulsed sinusoids is investigated for frequencies ranging between 0.15 and $12 \mathrm{kHz}$.

\section{METHOD}

\section{Apparatus}

The sinusoidal signals were $100 \mathrm{msec}$ in duration, gated on and off at positive-going zero crossings, and were generated by a Hewlett-Packard oscillator. Six frequencies were employed: $0.150,0.250,1,6,9$, and $12 \mathrm{kHz}$. Stimuli were bandpass filtered by two cascaded filters (Krohn-Hite 3550 ) centered at the frequency of the signal. The filters had a passband of $200 \mathrm{~Hz}$ and a combined attenuation rate of $48 \mathrm{~dB}$ per octave. Stimuli were presented binaurally over TDH-39 headphones.

\section{Procedure}

Masked thresholds and absolute thresholds were determined using a two-interval forced-choice procedure. The first of the two observation intervals, spaced $0.5 \mathrm{sec}$ apart, occurred $0.5 \mathrm{sec}$ after the start of the trial. In each observation interval, a standard tone of intensity I was presented. In one of the two observation intervals, an increment, $\Delta \mathrm{I}$, was added to the standard. The interval containing the increment was selected randomly. The listener indicated which observation interval he thought contained the increment by pressing one of two pushbuttons. The correct interval was indicated to the $O$ immediately after each response.

* This research was supported by a grant from the National Institute of Health and by the Human Resources Laboratory at Williams Air Force Base in Arizona.
Each session was divided into eight 15-min segments. Each segment, consisting of 140 trials, was devoted to determining a psychometric function for a given condition. An up-down psychophysical procedure was used for this purpose. The increment level varied from trial to trial over eight possible levels spaced $3 \mathrm{~dB}$ apart. The increment, therefore, ranged over $21 \mathrm{~dB}$. Each threshold increment was determined from an 8-point psychometric function. Except for the least and most attenuated signals, there were a minimum of 80 observations per point, since four psychometric functions were used to determine the increment corresponding to $P(C)=0.75$. At the extreme signal levels, however, there were only 40 observations per point. The psychometric functions were fitted by least-squares method to the equation $d^{\prime}=m(\Delta I)^{k}$ (Egan, Linder, \& McFadden, 1969).

Two sets of students attending Arizona State University served as listeners. All were paid $\$ 2 / \mathrm{h}$, except M.J.P., who is one of the authors. All conditions were randomized daily, and the Ss practiced about $6 \mathrm{~h}$ before any data were collected. The first set of three Os provided data for the masking function at frequencies from 0.150 to $9 \mathrm{kHz}$. The second set of two Os provided data for a replication of the $9-\mathrm{kHz}$ masking function and for a determination of the $12-\mathrm{kHz}$ masking function. In the replication, at the three most intense levels of the 9- and $12-\mathrm{kHz}$ tones, the psychometric function was determined nine times.

\section{RESULTS}

The masking functions for the first set of Os were obtained with the $0.150-, 0.250-, 1-, 6-$, and $9-\mathrm{kHz}$ tones and are displayed in Fig. 1. For each frequency, a least-squares technique was used to compute a linear fit to the masking data. Slopes for the individual data are presented in Table 1. The fitted function for the $1-\mathrm{kHz}$ condition is shown in Fig. 1 as a solid line. For all conditions and for all $\mathrm{Ss}$, the slope of the masking functions is nearly 0.9 .

The masking functions for the second set of Os were obtained with 9 - and $12 \mathrm{kHz}$ tones and are displayed in Fig. 2. For both frequencies, a least-squares technique was used to compute a linear fit to the masking data. Slopes for the individual data are presented in Table 2. The fitted function for the $9-\mathrm{kHz}$ condition for $\mathrm{S} 2$ is shown in Fig. 2 as a solid line. The linear fits were restricted to regions greater than $30 \mathrm{~dB}$ SL. It should 


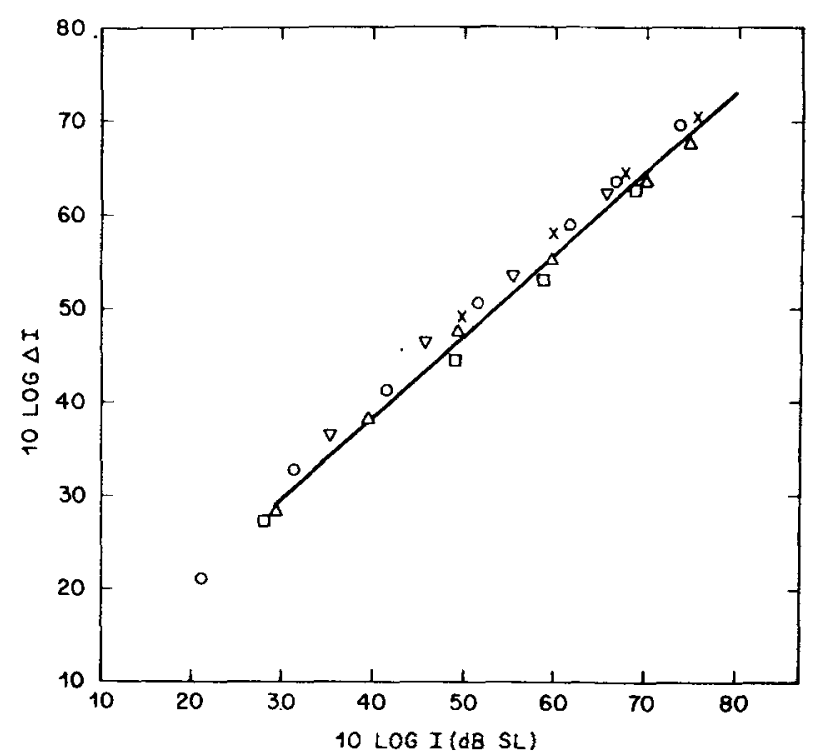

Fig. 1. Masking functions for pulsed sinusoids. On the abscissa is the level of the standard in dB SL. On the ordinate is the increment required for $75 \%$ correct detection in a two-interval forced-choice task. The upside-down triangles represent the data for the $150-\mathrm{Hz}$ tone, the squares, a $250-\mathrm{Hz}$ tone, the triangles, a $1-\mathrm{kHz}$ tone, the circles, a $6-\mathrm{kHz}$ tone, and the $\mathrm{Xs}$, a $9-\mathrm{kHz}$ tone. In order of increasing frequency, the absolute thresholds of the tones were $41,19,7,15$, and $16.5 \mathrm{~dB}$ SPL. The line is the least-squares fit to the $1-\mathrm{kHz}$ tone and has a slope of 0.86 .

Table 1

Slopes of the Masking Functions Estimated from a Least-Squares Fit of a Linear Function to the Data Shown in Fig. 1

\begin{tabular}{cccccc} 
& 150 & 250 & 1000 & 6000 & 9000 \\
\hline S 1 & - & 0.89 & 0.89 & 0.91 & - \\
S 2 & 0.87 & 0.89 & 0.85 & 0.84 & 0.82 \\
S 3 & 0.84 & 0.88 & 0.84 & 0.90 & 0.80 \\
Average & 0.86 & 0.89 & 0.86 & 0.88 & 0.81 \\
\hline
\end{tabular}

also be noted that the maximum level of the $12 \mathrm{kHz}$ tone was only $51 \mathrm{~dB}$ SL. It would obviously have been preferable to increase the level, but there were limitations imposed by the dynamic range of the equipment.

\section{DISCUSSION}

In agreement with previous data, the present results show that Weber's law fails to describe intensity discrimination for pulsed sinusoids in the region of $1,000 \mathrm{~Hz}$. Furthermore, this near-miss is independent of the signal frequency. Current theories of intensity discrimination must account for this finding. In the remainder of the discussion, three prominent theories of intensity discrimination are evaluated in light of the fact that Weber's law fails at all signal frequencies.

Zwicker $(1965,1970)$

Zwicker $(1965,1970)$ has explained the failure of
Weber's law in terms of a nonlinear growth in the "excitation" pattern caused by the stimulus. According to Zwicker, an increment can be detected whenever the excitation pattern changes by $1 \mathrm{~dB}$ or more. At high levels, the high-frequency side of the pattern grows more rapidly than the rest of the pattern. Thus, the 1-dB change necessary for detection would be produced by smaller $\mathrm{dB}$ increments than at low levels, independent of the frequency of the signal. Thus, Zwicker's model appears to predict a near-miss masking function that is independent of stimulus frequency. Our results are in accord with this prediction.

\section{McGill and Goldberg (1968a, b)}

McGill (1967) has proposed an elegant model of intensity discrimination that is based on the number of neural impulses reaching a counting center. The model assumes that the stimulus energy in an auditory channel is converted into neural impulses and that these impulses are collected at a counting center. A comparison of the total number of counts arising from samples of the standard alone and samples of the increment plus the standard forms the basis for the detection decision. An essential component of the model is the conversion of

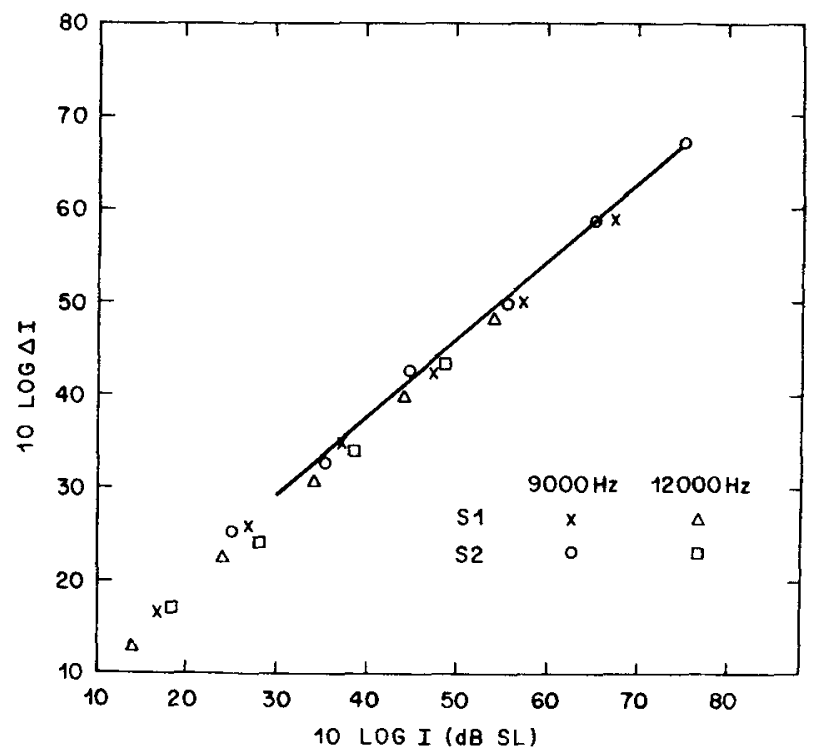

Fig. 2. Masking functions for pulsed sinusoids for a second set of Os. The sinusoidal frequency was $9 \mathrm{kHz}$ (Xs and circles) or $12 \mathrm{kHz}$ (triangles and squares). In order of increasing frequency, the abolute thresholds of the tones were 29 and $49 \mathrm{~dB}$ SPL. The line is the least-squares linear fit for $\mathrm{S} 2$ for the $9-\mathrm{kHz}$ tone and has a slope of $\mathbf{0 . 8 4}$.

Table 2

Slopes of the Masking Functions Estimated from a Least-Squares Fit of a Linear Function to the Data Shown in Fig. 2

\begin{tabular}{ccc} 
& 9000 & 12000 \\
\hline S 1 & 0.82 & 0.92 \\
S 2 & 0.84 & 0.92 \\
Average & 0.83 & 0.92 \\
\hline
\end{tabular}


the stimulus energy in the auditory channel into neural counts. In order to account for pure-tone intensity discrimination, McGill and Goldberg (1968a) posited a nonlinear mapping of stimulus energy into neural counts. In particular, McGill and Goldberg (1968a) concluded that the mean of the distribution of neural counts is related to stimulus energy by the following relationship:

$$
\text { Mean }=\mathrm{aE}^{\mathbf{p}},
$$

where $p$ is a number between 0 and $1, E$ is the energy in a single auditory channel, and a, a proportionality constant. Moreover, they show that the slopes of the function relating $\Delta \mathrm{I}$ and $\mathrm{I}$ on logarithmic coordinates are related to the parameter $p$ as follows:

$$
\text { Slope }=\frac{2-p}{2} \text {. }
$$

McGill (1967) has pointed out that the neural counting model is unable to predict changes in absolute threshold as a function of signal frequency. This does not appear to be a serious limitation since, as McGill notes, the constant a in Eq. 1 might be made to depend on the signal frequency. The utilization of the power-law relation introduces an additional possibility, namely, the exponent, $p$, could vary with signal frequency. The shape of the audiogram might imply that, for high-frequency tones, $p$ is smaller than it is for low-frequency tones. Although it is plausible that $p$ varies, the data presented here preclude it. The reasoning is as follows: The slope of the masking function is determined by $p$. The data presented in this paper demonstrated that the slope of the masking function does not depend on signal frequency. Hence, $p$ must remain constant across signal frequency. In order to account for the fact that signal threshold is a function of signal frequency, it must be the case that the constant, a, in Eq. 1 is not a constant, but rather depends on the signal frequency, as McGill (1967) has suggested.

\section{Viemeister (1972)}

Viemeister (1972) has suggested that Weber's law fails because of the use of information at the aural harmonics of the sinusoidal signal. He argues that if the intensity of the standard is sufficient, then a given change in the increment intensity produces a somewhat larger amplitude change at one or more of the aural harmonics. The change in level at the harmonic is the cue for detection. Thus, the Weber fraction decreases as the level of the standard increases. Consider the consequences of this rationale for the $9-\mathrm{kHz}$ sinusoid. The first aural harmonic of $9 \mathrm{kHz}$ is at $18 \mathrm{kHz}$. The data in Fig. 1 show that Weber's law fails for the $9-\mathrm{kHz}$ sinusoid. According to Viemeister, the failure of Weber's law means that the harmonic at $18 \mathrm{kHz}$ must be audible. However, it seems likely that the harmonic at $18 \mathrm{kHz}$ is not, in fact, audible. If so, then, according to Viemeister's explanation, the Weber fraction should remain constant and the masking function should have a slope to 1.0. The data do not support this conclusion.

\section{CONCLUSION}

In conclusion, we have found that the slope of the masking function is nearly 0.9 for $0.150-, 0.250-, 1-, 6-$, $9-$, and $12-\mathrm{kHz}$ sinusoids. These findings were discussed in terms of several theories of intensity discrimination. The failure of Weber's law at all signal frequencies appears - to agree with Zwicker's excitation pattern theory. In terms of McGill and Goldberg's model, the invariance of the slope of the masking function means that the $a$ in Eq. 1 is not a constant at all but, rather, depends on the signal frequency. Finally, the data for high-frequency tones do not appear to be in accord with Viemeister's (1972) postulate that information at the aural harmonics increases sensitivity and results in the failure of Weber's law.

\section{REFERENCES}

Campbell, R. A., \& Lasky, E. A. Masker level and sinusoidal-signal detection. Journal of the Acoustical Society of America, 1967, 42, 972-976.

Egan, J. P., Lindner, W. A., \& McFadden, D. Masking level differences and theform of the psychometric function. Perception \& Psychophysics, 1969, 6, 209-215.

McGill, W. J. Neural counting mechanisms and energy detection in audition. Journal of Mathematical Psychology, 1967, 4, 351-375.

MeGill, W. J., \& Goldberg, J. P. A study of the near-miss involving Weber's law and pure-tone intensity discrimination. Perception \& Psychophysics, 1968a, 4, 105-109.

McGill, W J. \& Goldberg, J. P Pure-tone intensity discrimination and energy detection. Journal of the Acoustical Society of America, $1968 \mathrm{~b}, 44,576-581$.

Viemeister, N. F. Intensity discrimination of pulsed sinusoids: The effects of filtered noise. Journal of the Acoustical Society of America, 1972, 51, 1265-1269.

Zwicker, E. Die elementaren Grundlagen zur Bestimmung der Informationskapazität des Gehörs. Acustica, 1965, 6, $365-381$.

Zwicker, E. Masking and psychological excitation as consequences of the ear's frequency analy sis. In R. Plomp and G. F. Smoorenburg (Eds.), Frequency analysis and periodicity detection in hearing. Leiden: Sifthoff, 1970.

(Received for publication June 29, 1973; revision received February 4,1974 .) 